\title{
Dinámica de microalgas frente a la costa de las Peñitas-Poneloya, Nicaragua
}

\author{
Microalgae dynamics and its relation with Las Peñitas Coast, Poneloya, Nicaragua
}

\author{
Sabrina Francisca Rojas Jirón ${ }^{1}$ \\ Joseyka Nayara Romero Peralta ${ }^{2}$ \\ Carmen Isabel Hernández Rivera ${ }^{3}$ \\ Noelia Erlinda Cea Navas ${ }^{4}$
}

\section{Resumen}

Esta es una investigación descriptiva que ha estudiado la dinámica de las microalgas (Cyanophytas, Chlorophytas, Bacillariophyceae y Dinoflagellata) y su relación con los parámetros físico-químicos de las costas de las Peñitas-Poneloya en el período de Junio-Octubre del 2017. Se tomaron muestras de 3 puntos diferentes entre el área comprendida en las coordenadas $12^{\circ} 20^{\prime} 10.63^{\prime \prime} \mathrm{N} 87^{\circ} 01^{\prime} 59.58^{\prime \prime} \mathrm{O}$ y $12^{\circ} 21^{\prime} 59.68^{\prime \prime} \mathrm{N}$ $87^{\circ} 04^{\prime} 13.27^{\circ} \mathrm{O}$. Estos muestreos se realizaban una vez al mes y en ellos se tomaban las muestras de agua y los parámetros físico-químicos ( $\mathrm{pH}$, salinidad, temperatura, turbidez y oxígeno). Se hacía conteo de células con ayuda de la cámara Neubauer y la identificación de microalgas con ayuda de la cámara SedgewickRafter. Se encontraron microalgas de los cuatro principales grupos, teniendo más representación de la clase Bacillariophyceae con 16 especies, seguidas de las Cyanophytas con 7 especies, Chlorophytas con 6 especies y Dinoflagellata con 5 especies. El parámetro de la salinidad oscilo entre $30 \mathrm{ppm}$ como valor mínimo presentado en el punto 1 y $34 \mathrm{ppm}$ como valor máximo presentado en el punto 3, la temperatura fluctuó entre $27^{\circ} \mathrm{C}$ y $29.7^{\circ} \mathrm{C}$, teniendo el valor mínimo en el mes de agosto en el 2do punto y la máxima en el mes de junio en el zer punto, la turbidez mantuvo un valor estable de $100 \mathrm{~cm}$ en sus 3 puntos durante todo el período de investigación. El oxígeno disuelto varió entre $7.3 \mathrm{mg} / \mathrm{l}$ y $8.3 \mathrm{mg} / \mathrm{l}$, teniendo su valor mínimo en el mes de junio en el 1er punto y el máximo en septiembre en su 1er punto. El pH osciló entre 8.6 su mínimo, encontrado en el mes de junio en el 2do punto y su máximo de 10.4 en el mes julio en el 2 do punto.

A todas las especies encontradas se le aplicaron los índices estadísticos Diversidad de Shannon y Dominancia de Simpson, para indagar si la zona cuenta con abundante biodiversidad o había dominancia de una especie específica, y según los resultados obtenidos se puede asegurar que el área cuenta con una diversidad y abundancia de especies de microalgas homogéneas.

Palabras clave: microalgas; dinámica; índice.

\section{Abstract}

This is a descriptive investigation that has studied the dynamics of microalgae (Cyanophytas, Chlorophytas, Bacillariophyceae and Dinoflagellata) and their relationship with the physical-chemical parameters of the Peñitas, Poneloya coasts in the period of June-October 2017. It was taken into account samples of 3 different points between the area included in the coordinates $12^{\circ} 20^{\prime} 10.63^{\prime \prime} \mathrm{N} 87^{\circ} 01^{\prime} 59.58^{\prime \prime} \mathrm{O}$ and $12^{\circ} 21^{\prime} 59.68^{\prime \prime} \mathrm{N} 87^{\circ} 04^{\prime} 13.27^{\prime \prime} \mathrm{O}$. These samples were carried out once a month to analyze water samples

\footnotetext{
1 Ingeniera Acuícola. Correo: sabrinajiron2018@gmail.com ORCID https://orcid.org/0000-0001-7872-4816

2 Ingeniera Acuícola. Correo romero.nayara95@gmail.com ORCID https://orcid.org/0000-0003-1745-0609

3 Master en desarrollo local sostenible, Profesora de la Escuela de Ciencias Agrarias y Veterinarias de la Universidad Nacional Autónoma de Nicaragua-León. Correo: carmen.hernandez@ev.unanleon.edu.ni ORCID https://orcid.org/0000-0002-3002-5255

4 Licenciada en Estadística. Profesor de la Escuela de Ciencias Agrarias y Veterinarias de la Universidad Nacional Autónoma de Nicaragua- León. Correo: noelia. cea@ev.unanleon.edu.ni ORCID: https://orcid.org/0000-0001-7913-9459
} 
and physical-chemical parameters ( $\mathrm{pH}$, salinity, temperature, turbidity and oxygen). Cell counts were done with the help of the Neubauer chamber and the identification of microalgae with the help of the Sedgewick-Rafter chamber. Microalgae of the four main groups were found, having more representation of the Bacillariophyceae class with 16 species, followed by Cyanophytas with 7 species, Chlorophytas with 6 species and Dinoflagellata with 5 species. The salinity parameter oscillated between $30 \mathrm{ppm}$ as the minimum value presented in point 1 and $34 \mathrm{ppm}$ as the maximum value presented in point 3 , the temperature fluctuated between $27^{\circ} \mathrm{C}$ and $29.7^{\circ} \mathrm{C}$, having the minimum value in the month of August in the second point and the maximum in the month of June in the third point, the turbidity maintained a stable value of $100 \mathrm{~cm}$ in its 3 points throughout the investigation period. The dissolved oxygen varied between 7.3 $\mathrm{mg} / 1$ and $8.3 \mathrm{mg} / \mathrm{l}$, having its minimum value in the month of June at the first point and the maximum in September at its first point. The $\mathrm{pH}$ ranged from 8.6 its minimum, found in the month of June in the second point and its maximum of 10.4 in the month of July in the second point.

The Diversity of Shannon and Dominance of Simpson statistical indexes were applied to all the species found, to inquire if the area has abundant biodiversity or there was dominance of a specific species, and according to the results obtained it can be ensured that the area has a diversity and abundance of homogeneous microalgae species.

Keywords: Microalgae; dynamics; index.

\section{Introducción}

Más del 80\% de la superficie terrestre está conformada por agua, esta a su vez se divide en agua dulce y salada. La investigación se realizó en cuerpos de agua salada, y se enfocó en el estudio de la dinámica de las microalgas (Cyanophytas, Chlorophytas, Bacillariophyceae y Dinoflagellata) y su relación con los parámetros físico-químicos de las costas de las Peñitas-Poneloya en el período de estudio de Junio-octubre del 2017. En este período se recolectaron muestras de agua que permitieran conocer la diversidad y abundancia de microalgas en esta zona, todo esto debido a que las microalgas son de suma importancia en los ecosistemas acuáticos ya que se estima que más del $85 \%$ de la fotosíntesis es realizada por estos micro organismos. De igual manera son el primer eslabón de la cadena trófica de los ecosistemas acuáticos, su importancia también recae en el aspecto económico sobre todo para el rubro camaronero. El área de investigación no poseía antecedentes investigativos enfocados en microalgas, y al poseer un alto potencial acuícola y pesquero, se realizó la investigación que permitió conocer la dinámica, abundancia y distribución de estas especies, también para conocer si el ambiente poseía especies de microalgas nocivas que pudieran afectar de manera directa la economía y salud de los habitantes de esa zona y de igual manera afectar la salud de los consumidores de los productos que se extraen de la zona (Lara 2008).

\section{Revisión de Literatura}

\section{Ecosistemas acuáticos}

La mayor parte de la superficie del planeta "Tierra” está cubierta de agua, estas a su vez se dividen en agua salada y dulce, ambas son capaces de sustentar la vida. Esta investigación se enfocó en el ecosistema de agua salada, en específico en las aguas costeras. Sin embargo, es de conocimiento general que todos los cuerpos de agua van a desembocar en las aguas marinas (Majluf, 2002).

Los sistemas acuáticos son muy dinámicos y presentan una conexión con corrientes superficiales y profundas. La temperatura y salinidad juegan el papel principal en la dinámica de las aguas, ya que estas permiten la formación de capas estratificadas, que pueden romperse por la surgencia de las aguas. Estos ecosistemas ocupan un papel importante para el desarrollo de la vida, para la determinación del clima y son el motor que transporta el calor y el agua dulce de la atmósfera (Lara, Arena Fuentes, Bazan Guzman, Díaz Castañeda, \& Escobar Briones, 2008). 
El mar, donde se originó la vida, posee una gran diversidad de ecosistemas; sin embargo, estos ecosistemas son poco conocidos, ya que hasta ahora, la biodiversidad terrestre es la más estudiada.

El mar, es heterogéneo y presenta varios tipos de ecosistemas que se clasifican según su zona de vida, como pelágicas asociadas a la superficie de las masas de agua, bentónica asociados a los fondos marinos, manglares, arrecifes de coral, etc. A su vez se diferencia las zonas costera, oceánica o marina, otra forma de clasificación es por la disponibilidad o ausencia de luz en eufótica y afótica. (Lara 2008).

\section{Fotosíntesis}

Es un proceso que realizan algunas células autótrofas para convertir sustancias orgánicas a inorgánicas. Esta es la manera que la energía lumínica se transforma en energía química, el Adenosin Trifosfato (ATP) es en donde la energía queda acumulada, estas moléculas de ATP sintetizan otras moléculas estables.

Este proceso es imprescindible para el planeta, ya que por medio de este es posible la fijación de Dióxido de Carbono ( $\mathrm{CO}_{2}$ ) y la consecuente liberación de oxígeno. Los factores que también influyen en este proceso son la temperatura, luminosidad y la concentración de Dióxido de Carbono en el ecosistema acuático (Hernández I. , 2014).

La fotosíntesis es realizada por micro y macro algas en los ecosistemas acuáticos en donde se consume una porción de la energía que es captada por el ecosistema. De este modo la fotosíntesis es la fuente de toda materia viva y de toda la energía biológica existente.

La reacción bioquímica de la fotosíntesis consta de dos fases consecutivas, la primera depende de las reacciones de la luz independiente de la temperatura (fase lumínica) y la otra depende de las reacciones de temperaturas independientemente de la luz (fase oscura), (Hernández I. , 2014).

\section{Fitoplancton}

Es un conjunto de organismos microscópicos fotosintetizadores que se encuentran en la columna de agua (Reynolds, 1984).

Las micro algas están compuestas en un 20 a 30\% de lípidos, de 40 a 50\% de proteínas y el sobrante entre carbohidratos que pueden alcanzar hasta el 55\% de biomasa en algunas microalgas (Borowitzka, 1988) Estas por su acción fotosintética pueden lograr beneficios solamente utilizando energía solar.

Debido a los diferentes lípidos y de otros compuestos poco comunes que pueden sintetizar las microalgas estas tienen la capacidad de desarrollarse en medios diferentes y desfavorables (Guschina \& Harwood, 2006) Dependiendo de la especie de microalga puede desarrollarse sobre un sustrato orgánico, con o sin presencia de luz (Travieso y Benitez, 1998).

Estos seres viven flotando en la columna de agua y son de origen vegetal, estos tienen un papel fundamental en la producción primaria. Generalmente son microscópicos y abundantes hasta en $200 \mathrm{mt}$ de profundidad aproximadamente. (Hernández I. , 2014)

Existen 4 tipos de alimentación de las microalgas; alimentación fotoautótrofa, alimentación foto-heterótrofa, alimentación mixotrófica y alimentación heterótrofa. En la alimentación fotoautótrofa, las microalgas producen su alimentos a través de la energía del sol y el carbono de compuestos inorgánicos; la alimentación fotoheterótrofa, se realiza cuando las microalgas reciben la energía del sol y utilizan compuestos orgánicos como fuente de carbono; alimentación mixotrófica, consiste en que las microalgas son capaces de crecer bajo procesos tanto autótrofos como heterótrofos, de manera que la fuente de energía es tanto la luz como la materia orgánica y el carbono lo obtienen tanto de compuestos orgánicos como 
inorgánicos, y la alimentación heterótrofa, reside en que los compuestos orgánicos proporcionan tanto la energía como la fuente de carbono. Es decir, existen algas que pueden crecer bajo ausencia de luz, como por ejemplo Chlorella protothecoides (Ruiz, 2011).

\section{Materiales y métodos}

El área de estudio comprendió $5 \mathrm{~km}$ dentro de las siguientes coordenadas $12^{\circ} 20^{\prime} 10.63^{\prime \prime} \mathrm{N} 87^{\circ} 01^{\prime} 59.58^{\prime \prime} \mathrm{O}$ y $12^{\circ} 21^{\prime} 59.68^{\prime \prime} \mathrm{N} 87^{\circ} 04^{\prime} 13.27^{\prime \prime} \mathrm{O}$, ubicadas a $2.5 \mathrm{~km}$ de la costa de Las Peñitas-Poneloya. Esta investigación es del tipo mixta, ya que combinan al menos un componente de los métodos cualitativos y cuantitativos.

Los muestreos se realizaron una vez al mes durante un período de 5 meses, además de fitoplancton se monitorearon parámetros físicos químicos. Para tomar las muestras se requirió de un muestreador de PVC de 2 pulgadas de diámetro y de $1.50 \mathrm{~m}$ de largo, una vez localizados en el primer punto de muestreo se procedió a sumergir el tubo en la columna de agua para recolectar la muestra de diferentes partes de la columna de agua (Zumaya Higuera, 2013).

Una vez recolectadas las muestras se colocaron en un recipiente, donde se homogenizaron las muestras de cada punto, una vez realizado este proceso se colocaron en 3 frascos plásticos de pruebas de $25 \mathrm{ml}$, donde se fijaron con solución Lugol, y se resguardaron en un termo para que no aumentaran su temperatura, ni se viera afectada por los rayos del sol. La muestra de agua se dejó reposar entre 18 y 24 horas para proceder a identificar y contabilizar a través del uso de la cámara de Neubauer y Sedwig-Rafter. (Molina., 2014).

EL análisis de datos estadísticos, se realizó mediante una tabla de Excel en donde se realizó la aplicación de las formulas correspondientes para obtener el Índice de diversidad y Abundancia de Shannon Wiener e Índice de Dominancia de Simpson, en donde se logró caracterizar las comunidades fitoplanctónica presentes durante el estudio.

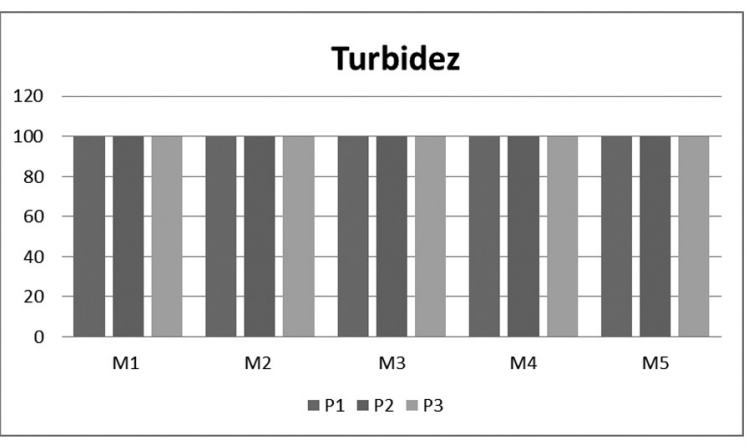

Figura $\mathrm{N}^{\circ}$ 3. Niveles de Turbidez de cada uno de los muestreos, correspondientes al M1 junio, M2 julio, M3 agosto, M4 septiembre, M5 octubre.

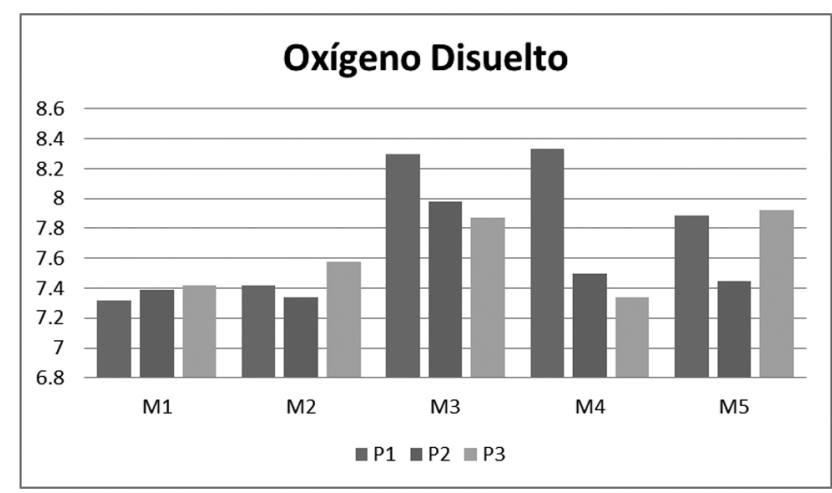

Figura $\mathrm{N}^{\circ}$ 4. Niveles de Oxígeno Disuelto de cada uno de los muestreos, correspondientes al M1 junio, M2 julio, M3 agosto, M4 septiembre, M5 octubre. 


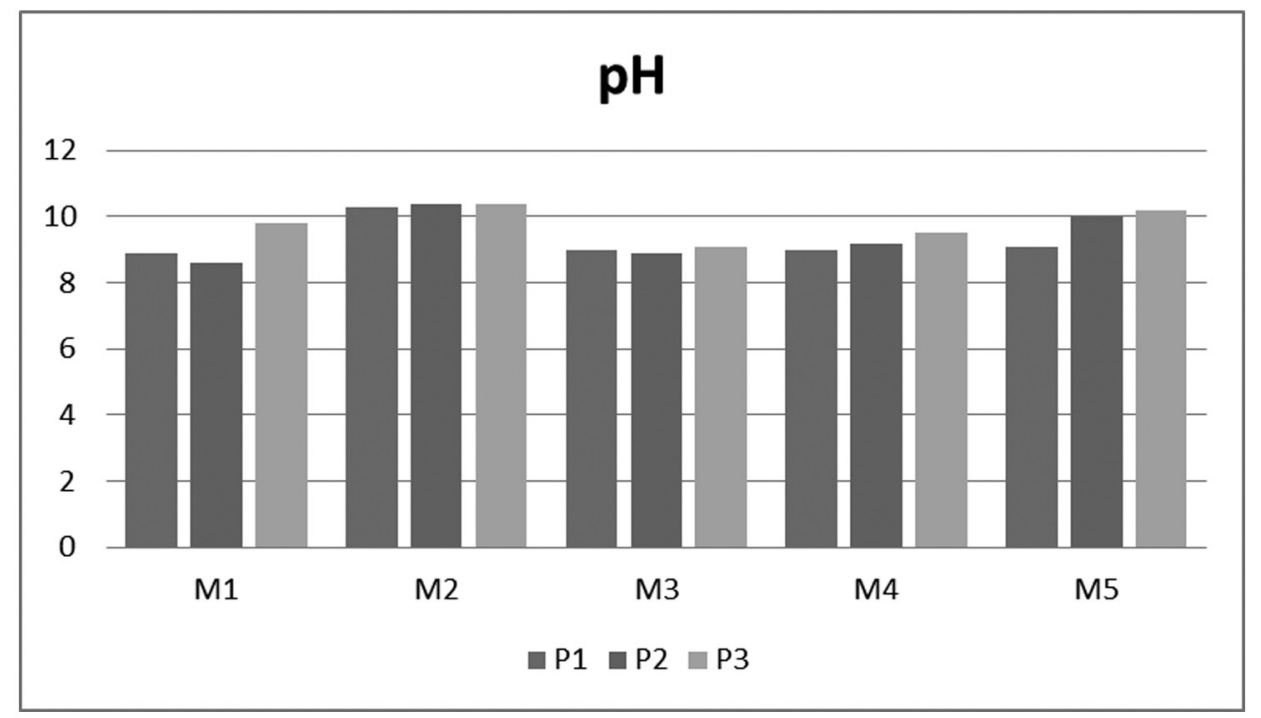

Figura $\mathrm{N}^{\circ}$ 5. Niveles de pH de cada uno de los muestreos, correspondientes al M1 junio, M2 julio, M3 agosto, M4 septiembre, M5 octubre.

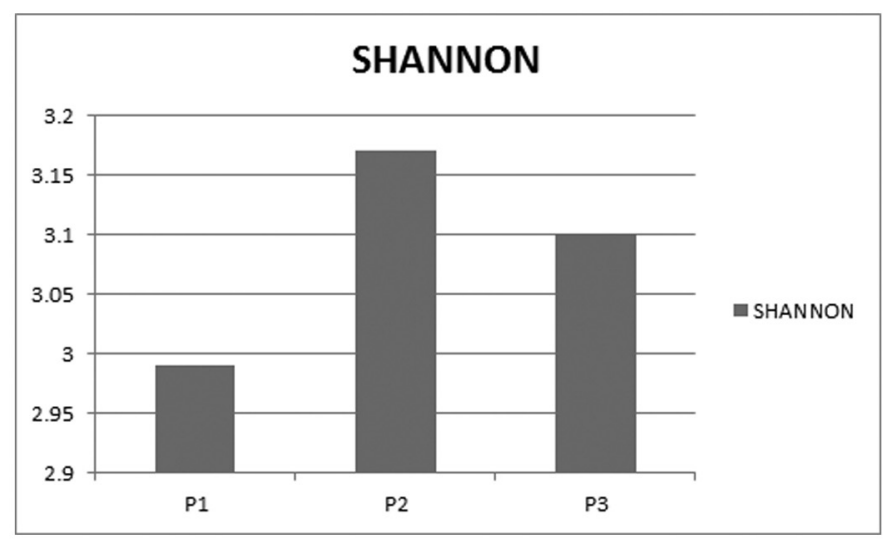

Figura $N^{\circ} 6$. Resultados del Índice de diversidad de Shannon -Wiener.

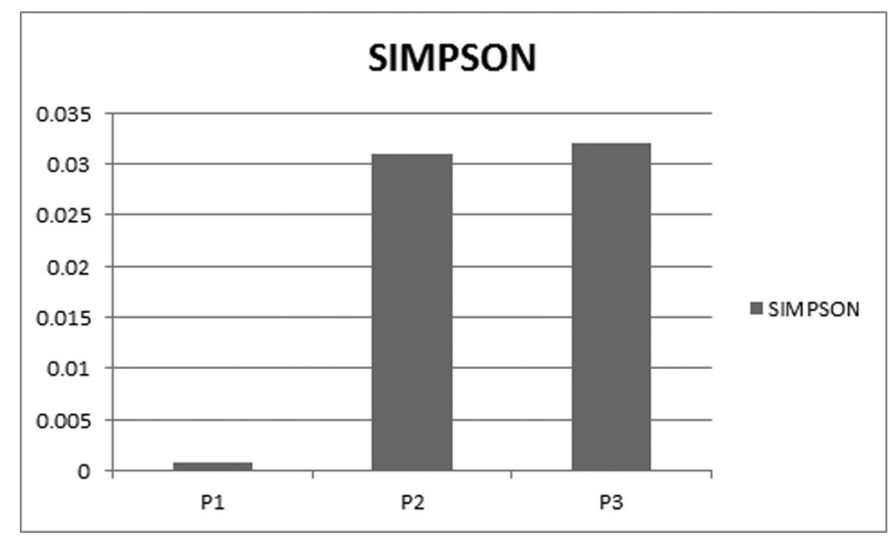

Figura № 7. Resultados de índice de Dominancia Simpson. 
Cuadro No. 1: Especies de microalgas encontradas.

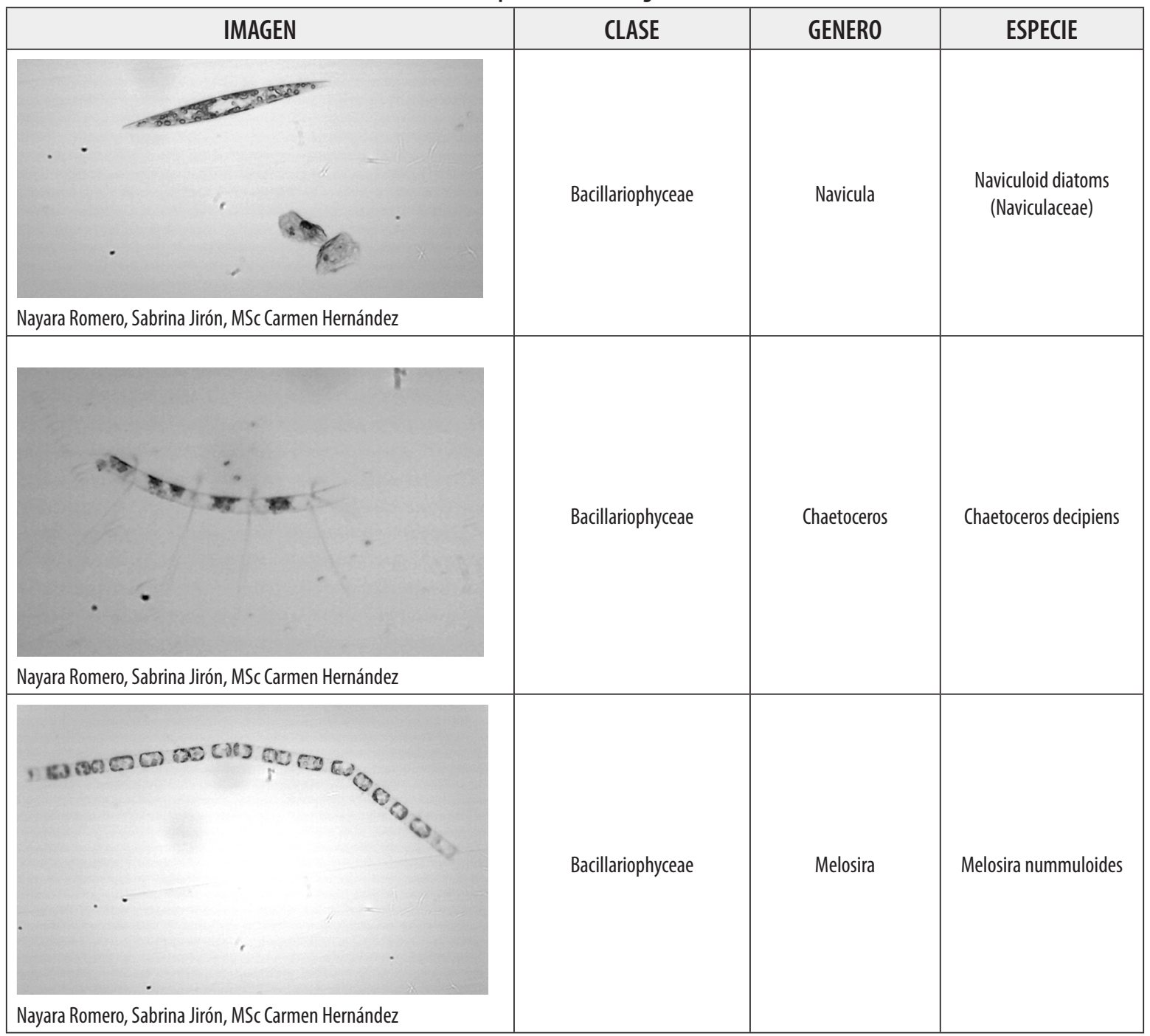

La figura $\mathrm{N}^{\circ}$ 1. Muestra los resultados obtenidos del parámetro de salinidad durante los 5 muestreos, en sus 3 puntos. Según Herrera (2012), la salinidad promedio del Pacífico es de 34.5-37 ppm. El dato más bajo encontrado durante la investigación fue de 30 ppm y el más alto de $34 \mathrm{ppm}$, lo que indica que estaban levemente bajo el rango establecido según bibliografía.

La composición salina de los océanos y el calentamiento global están variando de manera contemporánea, aumentando la evaporación atrapando calor en la atmósfera, reforzando el efecto invernadero entre un 5 y un $10 \%$ en la última década, provocando serias consecuencias en el ciclo del agua. Apareciendo estos cambios después de la década de los 90.

La alteración del parámetro se debió a que los muestreos fueron realizados en época de invierno, y generalmente había fuertes lluvias los días previos a los muestreos, lo que disminuían los iones salinos disueltos en el cuerpo de agua.

La figura $\mathrm{N}^{\circ} 2$ muestra los resultados de la temperatura obtenidos durante el periodo de investigación. Dando como dato máximo $29.7^{\circ} \mathrm{C}$ y mínimo $27^{\circ} \mathrm{C}$, estos resultados están encima del promedio optimo que es de $25^{\circ} \mathrm{C}$ (Majluf, 2002). 
El aumento de la temperatura, se debe a las emisiones de gases de efecto invernadero, que está ligada directamente con el cambio climático, y repercute en las aguas oceánicas, dando así altas temperaturas. Así mismo influyo la hora de la toma de parámetros, y la poca turbidez de la zona, ya que penetraban de manera directa los rayos solares.

Las actividades humanas que emiten dióxido de carbono atrapan el calor, generando un aumento anual de más del $0.6^{\circ} \mathrm{C}$ en los océanos, desde la superficie hasta unos $700 \mathrm{mt}$ de profundidad donde habitan la flora y fauna marina (National Geografhic, 2010).

El aumento de la temperatura de los océanos se ha considerado el mayor indicador de cambio climático ya que la capacidad calórica del océano es mil veces más fuerte que la actividad calorífica de la atmósfera.

Teniendo en cuenta que el océano es el termorregulador atmosférico, el aumento de temperatura conlleva a un transporte de calor hacia la atmósfera de manera continua.

Según Enriquez (2012), la turbidez en las agua marina oscila entre 100 y $130 \mathrm{~cm}$ de profundidad. Los resultados obtenidos fueron de $100 \mathrm{~cm}$ durante toda la etapa investigativa, manteniendose dentro de lo establecido por bibliografía. La turbidez fue tomada entre las 10 am y 2 pm que es cuando el sol se encuentra en su punto mas alto, lo que permitió tener un dato mas certero del parámetro.

El ángulo con que penetran los rayos solares en la capa de agua cambia a lo largo del día: En la mañana y a medio día la penetración es mucho mayor. La refracción solar también se modifica de acuerdo a la salinidad, teniendo mayor penetración cuando aumenta la concentración de sales.

La luz solar que logra penetrar en la capa de agua se transforma una parte en calor y la otra es utilizada por los microorganismos que viven en ella. La radiación solar es absorbida de maneras diferentes, las radiaciones rojas-naranjas son las que se absorben con mayor rapidez, mientras que las verde-azul son más lentas de procesar, es por eso que el agua de mar cambia entre colores azul oscuro y verde a lo largo de las costas.

En la figura $\mathrm{N}^{\circ} 4$ muestra los resultados obtenidos del parámetro Oxígeno (OD), dando como dato mínimo 7.3 mg/l y un máximo de $8.3 \mathrm{mg} / \mathrm{l}$. Majluf (2002) asegura que las aguas costeras de Pacífico oscilan entre 4.5 a $8 \mathrm{mg} / \mathrm{l}$, lo que indican que estaban dentro de los valores óptimos según bibliografía.

Herrera (2012), indica que el OD esta directamente relacionado con la temperatura, y que a mayor temperatura, menor oxígeno. Sin embargo, hay que recalcar que el océano es un cuerpo de agua inmenso, y que debido al efecto invernadero en los últimos años se han derivado cambios importantes, lo que significa que un aumento gradual en temperatura, no necesariamente repercutirá en el oxígeno. De igual manera las microalgas producen oxígeno mediante el proceso de la fotosíntesis lo que contribuye a mantener los niveles tal como se expresan en la figura 4.

Herrera (2012), indica que el pH óptimo en aguas costera es de 7.5 a $8.5 \mathrm{ppm}$, los rangos encontrados siempre estuvieron por encima de los valores óptimos, ya que el valor mínimo fue de $8.6 \mathrm{ppm}$ y el valor máximo de $10.4 \mathrm{ppm}$. Estos resultados se debieron al aumento de $\mathrm{CO}_{2}$ generado por el desarrollo de actividades antropogénicas, reflejado en el aumento de la temperatura. De igual manera las lluvias previas a los muestreos, causaban alteración en los iones de hidrogeno presentes en las aguas, dando como resultado un aumento excesivo del parámetro.

Cabe destacar que durante el día el fitoplancton consume dióxido de carbono y por ende el pH aumenta, ya que al disminuir el $\mathrm{CO}_{2}$ del ambiente, de igual manera lo hacen los iones hidrógeno, provocando el aumento de este. La acidificación puede causar una reducción del fitoplancton y afectar a especies que dependen de él para alimentarse. La acidificación de las aguas oceánicas debilita los procesos metabólicos 
de distintos organismos desde respiración hasta reproducción, pudiendo interrumpir en un futuro la producción, diversidad y resiliencia oceánica.

El efecto invernadero producto de las actividades antropogénica se debe principalmente al uso irracional de los recursos naturales, la tala y quema de bosques (que aumenta el $\mathrm{CO}_{2}$ del ambiente) son los principales causantes de los gases invernaderos, de igual manera las grandes industrias, ganadería, agronomía, que con la utilización de químicos (para control de plagas y enfermedades) y su arrastre por las lluvias, con su llegada a las aguas oceánicas alteran de manera considerable todas las características físico-químicas del agua. Forzando así la adaptación de las especies presentes.

El cambio climático impacta negativamente en las aguas oceánicas, alterando principalmente los parámetros fisicoquímicos y estos a su vez a las especies que en él habitan. Una de las principales consecuencias que puede haber es la floración de especies nocivas de microalgas (FAN), estas a su vez afectarán a los organismos acuáticos que se desarrollan en el medio afectado por la FAN y finalmente al ser humano cuando consume el producto.

Todo esto conlleva un proceso de cambios drásticos en el medio ambiente, produciendo alteraciones de los parámetros físico-químicos, que al estar todos relacionados, a la mínima desestabilización de uno se desata un desequilibrio en el habitad, y debido a las consecuentes alteraciones de los organismos y microorganismos, así como las microalgas, se ven obligadas a la adaptación del cambio o en su defecto a la disminución o terminación de la especie.

Por el momento las especies se van adaptando, pero de no frenar el efecto invernadero las consecuencias serán más graves, aumento del nivel de mar, migración de las especies, desplazamiento de los polos, contaminación por especies nocivas, etc.

Los resultados obtenidos, durante toda la investigación en su mayoría no coinciden con los datos consultados en bibliografías de crecimiento y distribuciones de microalgas; sin embargo, en la investigación se demuestra que aun fuera de los rangos establecidos pueden tener una abundancia superior al promedio y una perfecta distribución de las mismas. Lo que indica, que estos microorganismos van adaptándose a los cambios en los parámetros de su habitad, causados en su mayoría por el cambio climático. Manifestando con esta investigación que, debido a la adaptación de las microalgas en su medio natural, estas pueden reproducirse y sobrevivir fuera de los rangos que se han establecidos a lo largo de los años.

La diversidad y abundancia de microalgas en un ecosistema acuático es de suma importancia tomando en cuenta que este es la base de la cadena trófica de los ecosistemas acuáticos, en el estudio el índice de diversidad y abundancia de Shannon y Wiener se encontró entre 2.98 y 3.17, según Shannon (1949) el índice varía entre 0,5 y 5 , indicando que los valores inferiores de 2 son característicos de sistemas con baja abundancia, y superiores a 3 indican una alta abundancia y diversidad de especies. Tomando en cuenta lo descrito anteriormente se puede decir que los estudios fitoplanctónicos realizados en el área indican un ecosistema con presencia de abundante diversidad de microalgas.

Durante el estudio se calculó el Índice de Dominancia de Simpson que se mantuvo en un rango de o.001 y 0.033 estos datos nos corroboran lo indicado por el índice de Shannon y Wiener ya que no se presenta una dominancia de especie si no una diversidad de las mismas. Es importante mencionar que según Simpson (1949) cuanto más cerca de la unidad este, mayor es la probabilidad de dominancia de una sola especie, y cuanto más cerca del cero se encuentre se presenta menor dominancia tal como lo describen los resultados. 


\section{Conclusiones}

Los parámetros fisicoquímicos, a pesar que algunos no se mantuvieron dentro del rango óptimo según bibliografías, se logró demostrar que esto no fue limitante para el desarrollo de las microalgas en el cuerpo de agua.

Cada grupo de microalgas tiene diferentes rangos para crecimiento y reproducción; sin embargo, el $\mathrm{pH}$ es la mayor limitante de las mismas, inhibiendo la multiplicación de las células, debido a que el pH se encontró en valores de hasta 10.4, los conteos de células presentaron datos de $250,000 \mathrm{cel} / \mathrm{ml}$ en el mes de Julio y $62,500 \mathrm{cel} / \mathrm{ml}$ en el mes de octubre. Se encontraron un total de 34 especies de microalgas, distribuidos en 4 clases, predominando la clase Bacillariophyceae con 16 especies encontradas, a pesar de que las bibliografías consultadas indicaban parámetros exclusivos para el desarrollo y crecimiento de las especies, se demuestra a través de este estudio que las especies se están adaptando a los cambios ambientales que están sufriendo los ecosistemas acuáticos.

El índice de diversidad y abundancia de Shannon y Weaber se encontró entre 2.98 y 3.17, el índice de dominancia de Simpson se mantuvo en un rango de 0.001 y 0.033 , significando estos resultados que el área investigada es un habitad rico en diversidad de especies y abundancia de las misma.

\section{Agradecimiento}

Esta publicación obtuvo el financiamiento del Proyecto Ecosistemas Costeros, auspiciado por: Unión Europea, Dka Austria, Cooperación Austriaca para el Desarrollo y Horizontzooo.

\section{Lista de referencias}

Borowitzka, M. (1988). Grasas, aceites e hidrocarburos. Biotecnologia Microalgal. (Cambridge University Press.). Cambridge.

Enriquez, P. A. (2012). Manual de Biologia Pesquera. Obtenido de https://es.scribd.com/doc/108610734/ Manual-de-Biologia-Pesquera

Guschina, I. A., \& Harwood, J. (2006). Lipids and lipid metabolism in eukaryotic algae.

Hernandez, I. (2014). Manual de Fitoplancton. León, Nicaragua.

Herrera, C. (2012). Calidad de Agua. Leon., Nicaragua.

Lara, J. R., Arena Fuentes, V., Bazan Guzman, C., Díaz Castañeda, V., \& Escobar Briones, E. (2008). Los ecosistemas marinos: Conocimiento actual de la biodiversidad. Obtenido de http://www.biodiversidad. gob.mx/pais/pdf/CapNatMex/Vol I/Io5_Losecosistemasmar.pdf

Majluf, P. (2002). Los ecosistemas marinos y costeros. Obtenido de http://www.bio-nica.info/biblioteca/ Majluf2002.pdf

Molina., K. J. (2014). Dinámica del fitoplancton en las aguas del cultivo de la Isla Santa Lucia y el Laboratorio de investigaciones marina y acuícola (LIMA) en la comunidad Las Peñitas, León. Obtenido de http://riul. unanleon.edu.ni:8o8o/jspui/bitstream/123456789/3340/1/225248.pdf 
National Geografhic. (2010). National Geografhic. Obtenido de https:/www.nationalgeographic.es/ medio-ambiente/el-aumento-de-la-temperatura-del-mar

Reynolds, C. S. (1984). The Ecology of freshwater phytoplankton. . (C. U. ., Ed.) Cambridge., United Kingdom.

Ruiz, A. (2011). Puesta en marcha de un cultivo de microalgas para la eliminacion de nutrientes de un agua residual urbana previamente tratada anaerobicamente. (Universidad Politecnica de Valencia). España.

Shannon, C. a. (1949). Magmamater. Obtenido de http://www.magmamater.cl/MatheComm.pdf

Simpson, E. (1949). Nature. Obtenido de https://www.nature.com/articles/163688ao

Travieso, L., \& Benitez, F. (1998). Cultivo de Arthrospiras: del Laboratorio a Planta Piloto. Cur-so: Tecnología $y$ aprovechamiento del cultivo heterotrófico de microalgas. Centro de Investigaciones Pesqueras. La Habana, Cuba.

Zumaya Higuera, M. G. (2013). Aislamiento e identificación de microalgas marinas con potencial acuícola. Obtenido de https://www.google.com.ni/url?sa=t\&rct=j\&q=\&esrc=s\&source=web\&cd=3\&cad=rja\&uact=8\&ved=oahUKEwjZ_6nd5IHWAhVFKyYKHf7MA34QFgg1MAI\&url=http\%3A\%2F\%2Fbiblio.uabcs.mx\%2Ftesis\%2Fte3002.pdf\&usg=AFQjCNF4rVvzhdvp6Eta3kRGX8jzOP8tNw 